\title{
Sinergie e Sima, un ponte verso il futuro dell'Economia e Gestione delle Imprese
}

In molte riviste scientifiche gli editor si avvicendano con una certa frequenza. Sinergie nei suoi 37 anni di vita ha fatto eccezione con la direzione del fondatore Giovanni Panati fino alla sua scomparsa nel 1988, per poi vedere la direzione di Gaetano M. Golinelli affiancato nel 2009 da Claudio Baccarani.

Oggi la rivista si presenta ai suoi lettori con una nuova governance scientifica, a partire proprio dagli Editor in Chief con un processo di rinnovamento che coinvolge il Comitato Scientifico, gli Associate Editor, il Comitato di Redazione e prevede l'istituzione di un Comitato d'Onore.

In questa prospettiva anche la copertina è stata rivisitata abbandonando il tradizionale colore rosso per esprimere il cambiamento anche nella veste grafica.

Insomma, un mutamento nella rivista che rappresenta il frutto più tangibile di un dialogo e di una collaborazione strategica tra Sinergie e SIMA, la Società Italiana di Management, avviati nel 2016, attraverso il convegno annuale e condotti poi in un confronto sempre costruttivo sulle reciproche istanze.

A Sinergie e SIMA diffusa è la consapevolezza che nell'attuale fase agli studiosi di management è richiesto di porsi in modo compatto in dialogo con le istituzioni, le imprese, il Paese, le comunità scientifiche nazionali ed internazionali.

SIMA, con i suoi organi democraticamente eletti e forte del suo ruolo istituzionale e dei suoi progetti culturali, è il principale soggetto collettivo protagonista di tale dialogo; la rivista Sinergie Italian Journal of Management, con la comunità che da moltissimi anni la anima, si propone come punto di incontro e dibattito scientifico fondativo per l'identità degli studiosi italiani di management.

Ecco quindi che con questo numero 108, il primo numero del 2019, Sinergie si presenta come il journal ufficiale di SIMA e realizza un avvicendamento nella propria governance scientifica a partire dagli Editor in Chief. Gaetano M. Golinelli e Claudio Baccarani che lasciano la direzione e mettono il timone nelle mani di Marta Ugolini, come Editor in Chief, e di Alberto Pastore, come Co-Editor in Chief.

Il passaggio di consegne avviene nell'intento di mantenere viva la continuità con il passato di Sinergie e, al tempo stesso, di avviare percorsi innovativi e imprimere alla rivista sempre maggior apertura internazionale.

Con il supporto degli Associate Editor (di cui quattro nuovi) e del Comitato Scientifico, con la partecipazione attiva degli studiosi italiani di management e dei loro contatti internazionali, con l'apporto instancabile delle persone della Redazione, Sinergie vuole essere una rivista espressione 
sinergie Vol. 37, Issue 1, 2019

di una vera comunità, che porta avanti i valori della rilevanza unita al rigore, dell'ascolto, dellapertura a tutti gli studiosi, della semplicità nelle procedure e della valorizzazione delle relazioni umane, del lavoro condotto secondo fermi principi etici.

Una comunità che ambisce a generare delle ricadute positive per leconomia e la società nelle quali opera e che intende partecipare attivamente al dibattito della ricerca internazionale apportando il proprio contributo di conoscenza, mantenendo saldo il riferimento alle specificità del sistema produttivo e alle problematiche economico-manageriali del Paese.

Direttamente dalla voce dei protagonisti di questo avvicendamento, si possono trarre alcuni spunti sulla "nuova Sinergie".

Gaetano Golinelli ha dichiarato: "al neo direttore Marta Ugolini e al codirettore Alberto Pastore, che ho visto crescere scientificamente insieme a Sinergie, vanno gli auguri di un proficuo lavoro. Sono convinto che la loro solida esperienza, maturata nella rivista e in SIMA, saprà accrescere la rilevanza di Sinergie nel panorama scientifico italiano ed internazionale".

"Se penso alle origini della rivista ho difficoltà a percepire la dimensione del tempo trascorso - dice Claudio Baccarani. In effetti, un tempo così lungo mi si condensa in un attimo. Un "attimo" nel quale si sono susseguite tante entusiasmanti avventure che in modo quasi rocambolesco ci hanno portato sin qui. Sappiamo bene che il mondo dell'impresa attraverso le relazioni con lambiente in cui vive proporrà agli studiosi di management sempre nuove meravigliose ed emozionanti sfide. $\mathrm{E}$ in questo sempre sorprendente contesto sono certo che la rinnovata governance della rivista, con Marta Ugolini e Alberto Pastore nei loro ruoli di editor e co-editor, saprà trovare le vie per valorizzare la cultura d'impresa nel suo contributo al benessere comune".

Nel pensiero di Alberto Pastore "La partnership strategica tra SIMA e Sinergie, sul Convegno e sulla Rivista, è il frutto di un impegno reciproco fondato su solide basi. Da una parte sul senso istituzionale e sullo spirito di servizio a favore della nostra comunità di studiosi di management; dall'altra sul riconoscimento e sulla valorizzazione della nostra storia, coniugato alla volontà di restare protagonisti in uno scenario accademico in profondo cambiamento, nei suoi caratteri di qualificazione, rappresentatività, apertura e internazionalizzazione. In questo contesto, sono davvero orgoglioso di essere parte del progetto Sinergie Italian Journal of Management e onorato di poter proseguire il lavoro dei nostri Maestri. Sinergie Italian Journal of Management è la rivista di riferimento degli studiosi italiani, e può diventare il punto di incontro - anche a livello internazionale - per tutti coloro che guardano con interesse alle tematiche e ai caratteri distintivi delleconomia e del management del nostro Paese".

"Ė con gratitudine, consapevolezza dell'impegno assunto e una certa trepidazione - afferma Marta Ugolini - che raccolgo Sinergie dalle mani dei Maestri, Claudio Baccarani e Gaetano M. Golinelli. In questi anni ho contributo alla rivista come membro di una squadra, sempre con dei 
riferimenti forti. Oggi porterò avanti l'impegno con un peso maggiore di responsabilità. Non sono momenti semplici per una rivista autonoma dalle logiche dei grandi gruppi editoriali, specialmente internazionali. Editoriale primo numero

Sinergie e Sima, un ponte verso il futuro dell'Economia Non è forse il posizionamento come rivista generalista quello più adatto a collocarsi negli attuali mainstream di ricerca. Ma nel contesto ci sono indizi di cambiamento e del resto da Claudio Baccarani, più di ogni altro, ho imparato il valore dei sogni e delle sfide". 